\title{
Influence of Deuterium Retention on Secondary Electron Emission from Graphite under Deuterium Plasma Exposure
}

\author{
Tatsuya KANEKO, Noriyasu OHNO, Yuki NAKAMURA, Masato YAMAGIWA, \\ Noriaki MATSUNAMI ${ }^{1)}$, Shin KAJITA ${ }^{1)}$ and Makoto TAKAGI \\ Graduate School of Engineering, Nagoya University, Nagoya 464-8603, Japan \\ ${ }^{1)}$ EcoTopia Science Institute, Nagoya University, Nagoya 464-8603, Japan
}

(Received 2 September 2014 / Accepted 25 January 2015)

\begin{abstract}
The influence of deuterium retention on the electron-impact secondary electron emission (SEE) is studied in isotropic graphite (ETU-10). The ETU-10 surface sheath voltage and its deuterium retention under deuterium plasma exposure were measured simultaneously. Deuterium retention was estimated using in situ nuclear reaction analysis. While deuterium retention increased with decreasing graphite sample temperature, the sheath voltage on the sample surface decreased. The sheath potential variation is considered to be due to the SEE yield variation, which was estimated using the sheath voltage. The estimated SEE yield value increased by approximately $10 \%$ as the deuterium retention rose by a factor of two.
\end{abstract}

(C) 2015 The Japan Society of Plasma Science and Nuclear Fusion Research

Keywords: graphite, deuterium retention, secondary electron emission, ion beam analysis

DOI: $10.1585 /$ pfr. 10.1402009

\section{Introduction}

Carbon-based materials have been widely used for plasma-facing components in fusion devices such as LHD and JT-60U and will be used in JT-60SA because of their high heat load resistance and lower radiation energy loss. However, carbon-based materials can accumulate high concentrations of hydrogen isotopes [1-4], which influence plasma density control and tritium inventory in a future fusion reactor.

Furthermore, the previous study by K. Shiraishi et al. [5] suggested that the retention of hydrogen isotopes in carbon-based materials increases the secondary electron emission (SEE) from material surfaces. The increase in SEE leads to a decrease in plasma sheath voltage formed on the material surface [6]. The sheath voltage $\phi_{\mathrm{sh}}$ and the power transmission factor $\delta$ are given by [7]

$$
\begin{aligned}
& \frac{\phi_{\mathrm{sh}}}{T_{\mathrm{e}}}=\frac{1}{2} \ln \left\{\frac{2 \pi m_{\mathrm{e}}}{m_{\mathrm{i}}}\left(1+\frac{T_{\mathrm{i}}}{T_{\mathrm{e}}}\right) \frac{1}{(1-\sigma)^{2}}\right\}, \\
& \delta=\frac{2 T_{\mathrm{i}}}{T_{\mathrm{e}}}+\frac{2}{1-\sigma}-\frac{1}{2} \ln \left\{\frac{2 \pi m_{\mathrm{e}}}{m_{\mathrm{i}}}\left(1+\frac{T_{\mathrm{i}}}{T_{\mathrm{e}}}\right) \frac{1}{(1-\sigma)^{2}}\right\},
\end{aligned}
$$

where $m_{\mathrm{e}}$ is the electron mass, $m_{\mathrm{i}}$ and $T_{\mathrm{i}}$ are the ion mass and temperature, respectively, and $\sigma$ is the yield of secondary electrons including reflected primary electrons. $\delta$ is defined as the total incident electron and ion energy through the sheath to the wall, normalized by the product of the electron temperature and the ion flux. Figure 1 shows normalized sheath voltage and $\delta$ of deuterium plasma calculated by Eqs. (1) and (2) assuming $T_{\mathrm{i}} / T_{\mathrm{e}}=0$.

author'se-mail: ohno@ees.nagoya-u.ac.jp

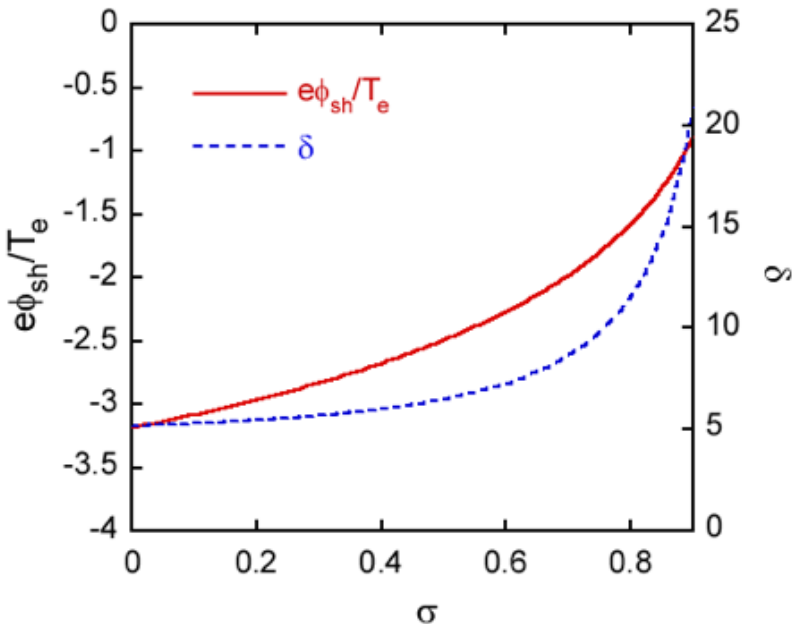

Fig. 1 SEE yield dependencies of sheath voltage normalized to the electron temperature (solid line) and power transmission factor (dotted line).

The decrease in the sheath voltage causes the enhancement of electron flux to a material surface, and consequently, the heat load to the material will increase. Therefore, hydrogen isotope retention should be an important factor to determine the heat load of plasma-facing components composed of carbon-based materials.

However, no previous studies concerning the relation between hydrogen isotope retention and SEE in carbon materials have been conducted during plasma exposure. Therefore, this study focuses on the influence of deuterium retention on the SEE characteristics during plasma 
exposure. The study was conducted using plasma surface dynamics with ion beam analysis (PS-DIBA) device [8], which makes it possible to conduct in situ ion beam analysis of deuterium retention.

\section{Experimental}

PS-DIBA consists of a compact and powerful DC plasma source and ion beam equipment. The plasma source is composed of $\mathrm{a} \mathrm{LaB}_{6}$ cathode and a copper anode and can produce a deuterium plasma with high flux of approximately $10^{22} \mathrm{~m}^{-2} \mathrm{~s}^{-1}$. The power source supplies the discharge power (up to $2 \mathrm{~kW}$ ), and two magnetic field coils produce a magnetic field with strength $B$ of up to $0.14 \mathrm{~T}$. Plasma parameters are measured by a fast scanning Langmuir probe made of tungsten.

We measured the deuterium retention by nuclear reaction analysis (NRA) using a Van de Graaff accelerator. For the $\mathrm{D}\left({ }^{3} \mathrm{He}, \mathrm{p}\right) \alpha$ NRA analysis, we used a mass-separated ${ }^{3} \mathrm{He}^{+}$ion beam with a primary energy of $1.0 \mathrm{MeV}$. The solid-state semiconductor detectors for NRA were set at a detection angle of $135^{\circ}$. They were protected by thin Mylar films $(6 \mu \mathrm{m})$ and aluminum foils $(12 \mu \mathrm{m})$ to repel plasma (neutral) particles and photons. The detector chamber was evacuated by a turbomolecular pump and kept below $10^{-4} \mathrm{~Pa}$ by using the Mylar film during plasma exposure. Since the ion beam current at the sample is difficult to measure during plasma exposure, we used a rotating gold plate located in the beam line, as shown in Fig. 2, to monitor the beam current. The beam current at the sample and the backscattering yield of the monitor were measured without plasma before the plasma exposure experiment. The amount of retained deuterium was determined by integrating the measured deuterium concentration over the entire detection depth (approximately $2.5 \mu \mathrm{m}$ ) with an uncertainty of $10 \%$. The ion beam was collimated at the sample to a size of approximately $2 \mathrm{~mm} \times 1 \mathrm{~mm}$, and the acquisition time was $30 \mathrm{~min}$.

The sample used was isotropic graphite ETU-10 ( $\phi 30 \mathrm{~mm} \times 5 \mathrm{~mm}$, IBIDEN CO., LTD). It was held on

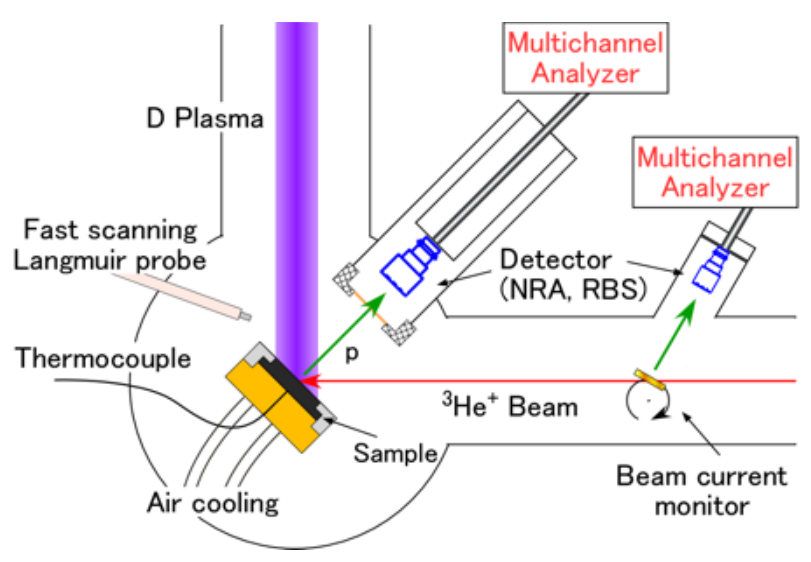

Fig. 2 Schematic view of the experimental setup in PS-DIBA. a sample manipulator and not biased during the experiment; the potential of the sample was kept floating electrically. The sample temperature was monitored by chromelalumel thermocouple placed on the rear surface of the sample. During the NRA measurement, the temperature was kept constant with an uncertainty of $5 \mathrm{~K}$.

\section{Results and Discussion \\ 3.1 Measurements}

First, the graphite sample was heated up to $550 \mathrm{~K}$ by deuterium plasma with a discharge current of $30 \mathrm{~A}$ and a gas pressure of 1.0 Pa. While changing the surface temperature (by changing the flow rate of air for the cooling stage), the deuterium retention in the sample was measured at 550, 500, 450, 400, and $350 \mathrm{~K}$ by NRA. Figure 3 shows the dependence of $\mathrm{D} / \mathrm{C}$ ratio on the sample temperature. The $\mathrm{D} / \mathrm{C}$ ratio increased from $0.22 \%$ to $0.57 \%$ with decreasing sample temperatures from 350 to $550 \mathrm{~K}$. Note that the $\mathrm{D} / \mathrm{C}$ ratio is determined by the ratio of the total number of measured deuterium atoms over the detection depth (approximately $2.5 \mu \mathrm{m}$ ) of NRA to that of carbon atoms at a depth of up to $2.5 \mu \mathrm{m}$.

To confirm the plasma stability, the time evolution of the floating potential of the probe $\phi_{\mathrm{fp}}$ and the electron temperature $T_{\mathrm{e}}$ were measured, as shown in Figs. 4 (a) and (b), respectively. Figure 4 (a) shows the measured $\phi_{\mathrm{fp}}$ values and a curve fitted by the least-squares method, which is represented by the markers and a solid line, respectively. Note that the floating potential rises in time, whereas $T_{\mathrm{e}}$ is almost constant. This experimental result indicates that plasma space potential $\phi_{\text {space }}$ changed with time probably due to the variation of DC discharge conditions. This effect should be considered in future analyses.

Figures 5 (a) and (b), respectively, show the time evo-

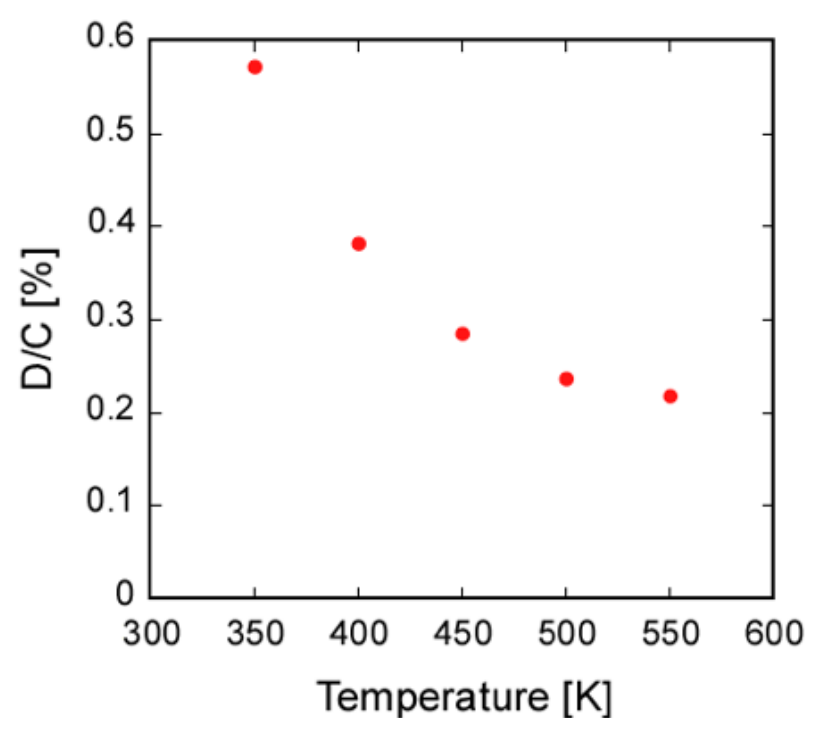

Fig. 3 Temperature dependence of $\mathrm{D} / \mathrm{C}$ ratio of the graphite sample. 

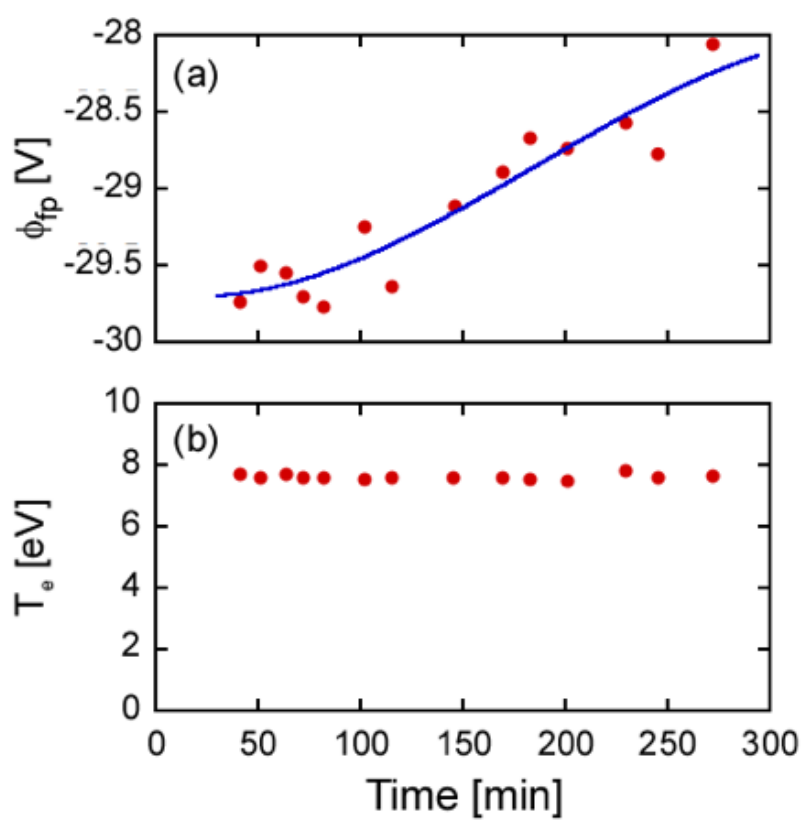

Fig. 4 (a) Floating potential of probe and (b) electron temperature measured by the fast scanning Langmuir probe.
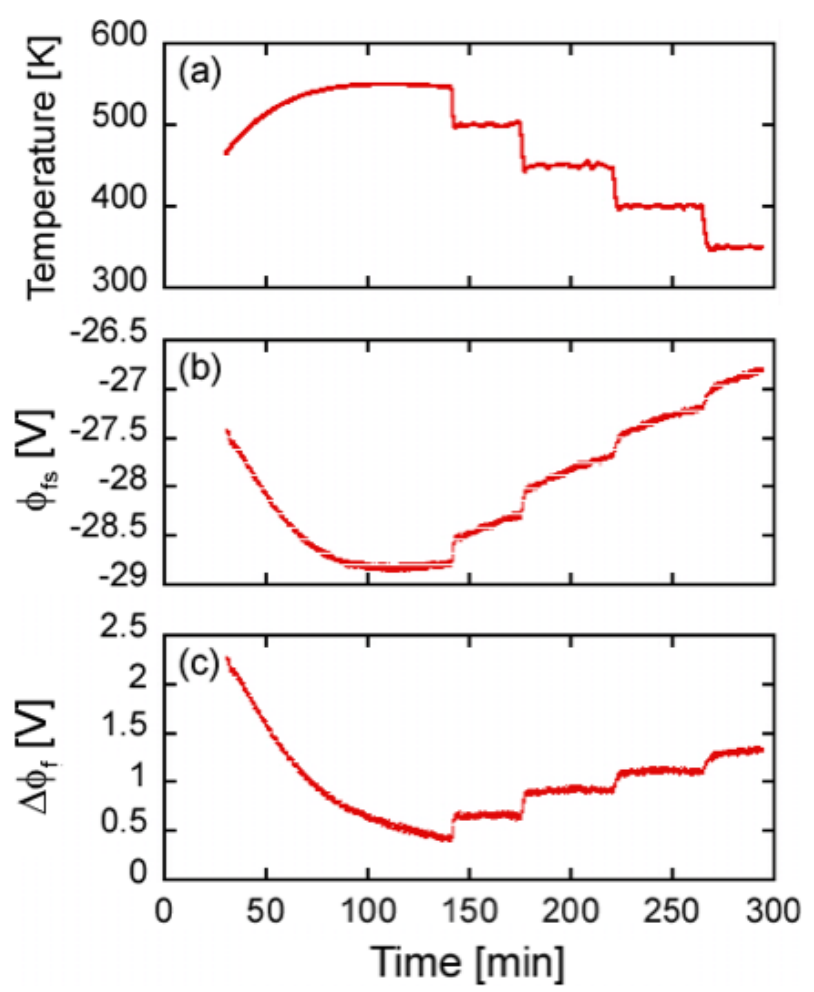

Fig. 5 Time variation in (a) sample temperature, (b) floating potential of sample, and (c) the difference in floating potential between the sample and probe.

lutions of the sample temperature measured by thermocouple and the floating potential of the sample $\phi_{\mathrm{fs}}$. The change in the floating potential at each temperature is attributed to the change in the space potential, as is the case in that of the probe mentioned above.

The floating potential depends on the plasma space potential, the potential drop in the pre-sheath, and the sheath voltage. The sheath voltage $\phi_{\mathrm{sh}}$ is given by

$$
\phi_{\mathrm{sh}}=\frac{T_{\mathrm{e}}}{2} \ln \left\{\frac{2 \pi m_{\mathrm{e}}}{m_{\mathrm{i}}}\left(1+\frac{T_{\mathrm{i}}}{T_{\mathrm{e}}}\right) \frac{1}{(1-\sigma)^{2}}\right\} .
$$

The SEE yield of the probe is expected to be constant because the probe temperature and the deuterium retention in the probe appeared to be approximately constant during this series of experiments. In addition, as shown in Fig. 4(b), the electron temperature $T_{\mathrm{e}}$ was approximately constant in this experiment. Thus, the sheath voltage and the potential drop in the pre-sheath near the probe surface, which are proportional to the electron temperature, were considered to be constant.

Figure 5 (c) shows the difference between the floating potential at the probe and the sample. The difference is approximately constant at each sample temperature, which is consistent with the fact that the change in the floating potential was due to the change in the space potential.

\subsection{SEE yield estimation}

In this experiment, since the sample surface was much larger than that of the probe and the space potential had a radial profile, the space potentials of the sample and probe could be different even without the difference in SEE. Considering the difference in the space potentials, $\Delta \phi_{\mathrm{f}}$ in Fig. 5 (c) can be expressed by the differences in the sheath potential of Eq. (3) and the space potentials at the positions of the sample and the probe. Thus, $\Delta \phi_{\mathrm{f}}$ is expressed by

$$
\Delta \phi_{\mathrm{f}}=T_{\mathrm{e}} \ln \left|\frac{1-\sigma_{\mathrm{W}}}{1-\sigma_{\mathrm{C}}}\right|+\Delta \phi_{\text {space }}
$$

where $\sigma_{\mathrm{W}}$ and $\sigma_{\mathrm{C}}$ are the SEE yields of tungsten (probe tip material) and graphite, respectively, and $\Delta \phi_{\text {space }}$ is the difference in the space potentials. Using the above expression, $\Delta \phi_{\mathrm{f}}$ variations and $\sigma_{\mathrm{C}}$ as the sample temperature varied from $T_{0}$ to $T$ are expressed as follows:

$$
\begin{aligned}
& \Delta \phi_{\mathrm{f}}-\Delta \phi_{\mathrm{f} 0}=T_{\mathrm{e}} \ln \left|\frac{1-\sigma_{\mathrm{C} 0}}{1-\sigma_{\mathrm{C}}}\right| \\
& \sigma_{\mathrm{C}}=1-\left(1-\sigma_{\mathrm{C} 0}\right) \exp \left(-\frac{\Delta \phi_{\mathrm{f}}-\Delta \phi_{\mathrm{f} 0}}{T_{\mathrm{e}}}\right),
\end{aligned}
$$

where $\sigma_{\mathrm{C} 0}$ and $\Delta \phi_{\mathrm{f} 0}$ are the SEE yield and the difference in the floating potential at the sample temperature of $T_{0}$, respectively. From the above equations, $\sigma_{\mathrm{C}}$ was obtained, as shown in Fig. 6(a), using the difference in the floating potential at a sample temperature of $500 \mathrm{~K}$ as $\Delta \phi_{\mathrm{f} 0}$, for $\sigma_{\mathrm{C} 0}$ values of $0.3,0.4$, and 0.5 . In addition, for the cases of no difference between the space potentials at the positions of the sample and the probe, $\Delta \phi_{\mathrm{f}}$ equals the difference between the sheath voltage of the sample and the probe, and 

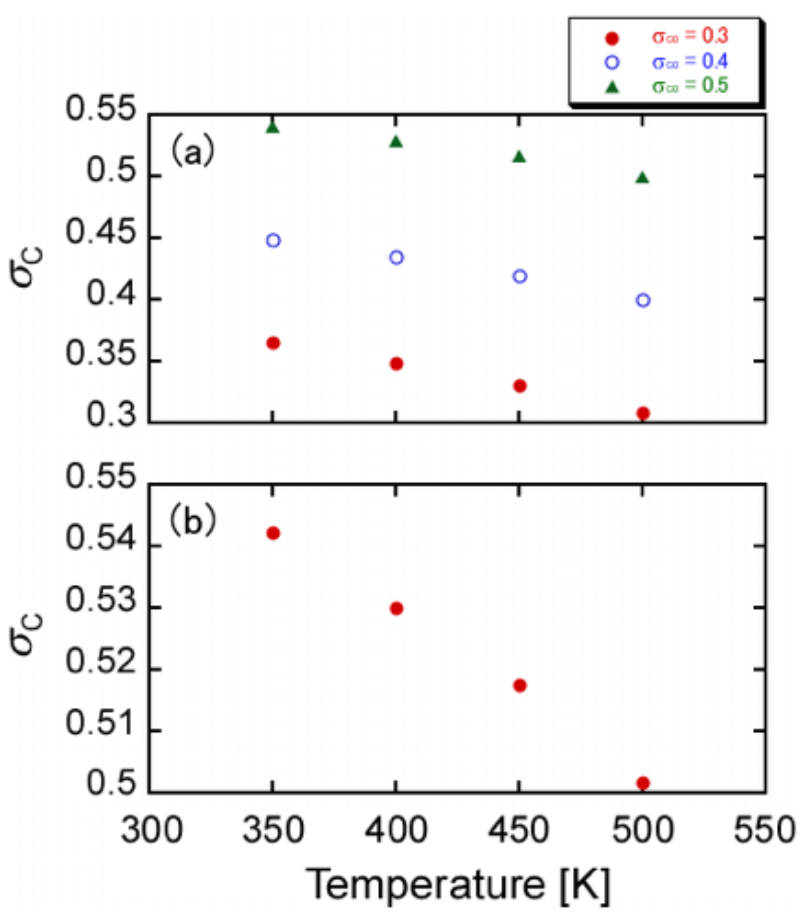

Fig. 6 Temperature dependence of SEE yield of graphite sample (a) calculated by Eq. (6) and (b) Eq. (8).

$\Delta \phi_{\mathrm{f}}$ and $\sigma_{\mathrm{C}}$ are expressed as follows:

$$
\begin{aligned}
& \Delta \phi_{\mathrm{f}}=T_{\mathrm{e}} \ln \left|\frac{1-\sigma_{\mathrm{W}}}{1-\sigma_{\mathrm{C}}}\right|, \\
& \sigma_{\mathrm{C}}=1-\left(1-\sigma_{\mathrm{W}}\right) \exp \left(-\frac{\Delta \phi_{\mathrm{f}}}{T_{\mathrm{e}}}\right) .
\end{aligned}
$$

Using a value of $\sigma_{\mathrm{W}}=0.46$ given by the Monte Carlo simulation [9], $\sigma_{\mathrm{C}}$ in the above expression is obtained, as shown in Fig. 6 (b). The SEE yield increased as the sample temperature decreased, as shown in Figs. 6 (a) and (b). $\sigma_{\mathrm{C}}$ variation with the sample temperature was smaller for large $\sigma_{\mathrm{C} 0}$.

Figures 7 (a) and (b), respectively, show the plots of the SEE yields in Figs. 6 (a) and (b) against the deuterium retention. The SEE yields increased with the deuterium retention, which is consistent with the previous studies $[5,10]$. In Fig. 7 (b), the increase in the SEE yield is approximately $10 \%$, whereas the deuterium retention increased by more than twofold. Furthermore, the increase in the SEE yield shown in Figs. 7 (a) and (b) appears to become saturated with the increase in the deuterium retention.

To compare the estimated SEE yield in Fig. 7 (b) with the previous experimental estimates is slightly difficult since the previous experiments for electron-impact SEE were conducted using high-energy electron beams of more than $20 \mathrm{eV}$ as primary electrons. J.M. Pedgley and G.M. McCracken reported that the SEE yield for clean graphite at a primary electron energy of $20 \mathrm{eV}$ was approximately 0.45 [11]. For graphite implanted with $3 \mathrm{keV} \mathrm{H}_{2}^{+}$ions to

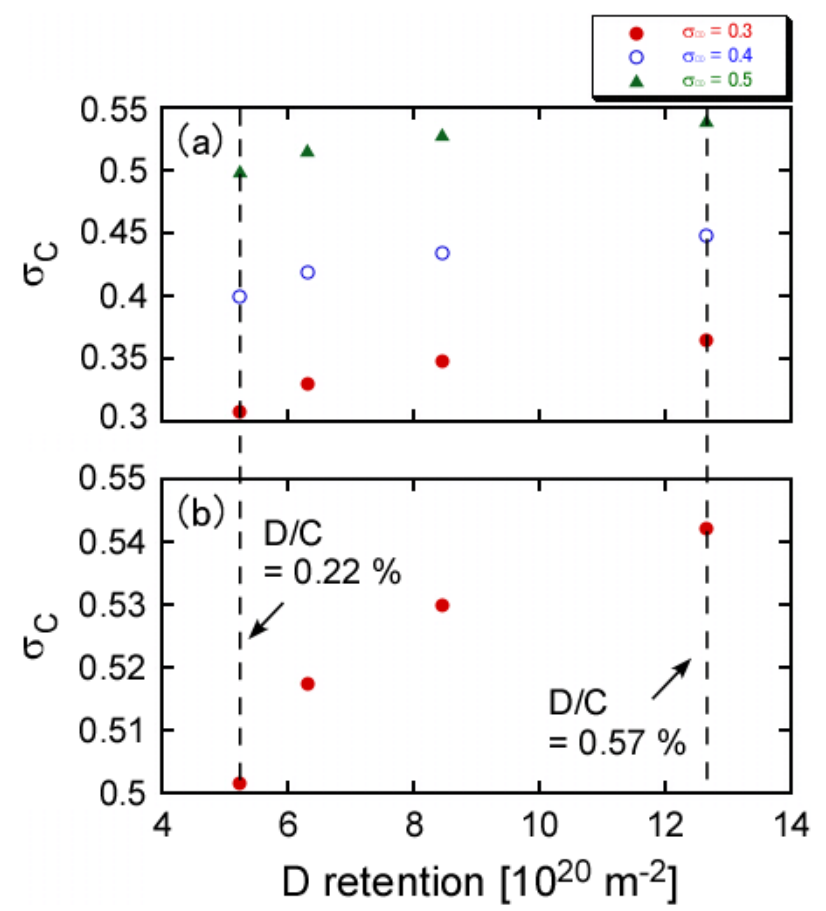

Fig. 7 Deuterium retention dependence of SEE yield of graphite sample (a) calculated by Eq. (6) and (b) Eq. (8).

a dose of $6.4 \times 10^{17}$ ions $\mathrm{cm}^{-2}$, the SEE yield was enhanced to 0.48 . In Ref. [11], there was no information about retained hydrogen. Under our experimental conditions, the typical dose of deuterium ions was more than $10^{21}$ ions per $\mathrm{cm}^{2}$, which is much higher than the dose used in Ref. [11]. Furthermore, dynamically retained deuterium atoms exist near the surface under deuterium plasma exposure $[12,13]$. The number of retained deuterium atoms near the surface should be much larger than that in Ref. [11], which should lead to a further increase in the SEE yield. Conversely, the electron temperature was $7.7 \mathrm{eV}$ in our experiment, which does not correspond to a mono energy, but the averaged electron energy is smaller than $20 \mathrm{eV}$. Such a low electron impact energy should reduce the SEE yield. Therefore, the SEE yield of approximately 0.5 in Fig. 7 (b) appears to be a reasonable value in comparison with that of Ref. [11].

The SEE yield for clean graphite was calculated using the Monte Carlo simulation [9]. The calculation provides an SEE yield of 0.31 at an electron temperature of $7.7 \mathrm{eV}$, corresponding to the experimental conditions. Moreover, this result supports the argument that the SEE yield increased because of the deuterium retention in our experiment.

\section{Conclusion}

The influence of deuterium retention on SEE has been investigated during deuterium plasma exposure on isotropic graphite (ETU-10). Deuterium retention was 
measured by in situ NRA during the deuterium plasma discharge in PS-DIBA. During the NRA measurement with 30-min acquisition time, the sample temperature was fixed by air cooling at 550, 500, 450, 400, and $350 \mathrm{~K}$. While deuterium retention increased with decreasing sample temperatures, the floating potential of the sample rose. Since this floating potential rise was due to the change in the plasma space potential, the sheath voltage of the graphite sample surface was estimated by considering the change in the space potential. Consequently, the sheath voltage was constant at constant sample temperature and increased with decreasing sample temperatures.

The sample SEE yield was estimated based on the sheath voltage variation. The estimated SEE yield increased with deuterium retention, which is consistent with previous studies. Furthermore, the increase in the SEE yield appears to become saturated with increasing deuterium retention.

[1] R.A. Causey and K.L. Wilson, J. Nucl. Mater. 138, 57 (1986).
[2] K. Morita, K. Ohtsuka and Y. Hasebe, J. Nucl. Mater. 162164, 990 (1989).

[3] B. Emmoth, H. Bergsaker and L. Ilyinsky, J. Nucl. Mater. 241-243, 1022 (1997).

[4] A. Rusinov, N. Trifonov et al., J. Nucl. Mater. 417, 616 (2011).

[5] K. Shiraishi et al., J. Plasma Fusion Res. 69, 1371 (1993).

[6] K. Ohya, K. Nishimura et al., J. Nucl. Mater. 196-198, 699 (1992).

[7] P.C. Stangeby, Physics of Plasma-Wall Interactions in Controlled Fusion ed. by D.E. Post and R. Behrisch, NATO ASI Series B: Physics Vol.131 (Plenum Press, New York, 1986) p.41.

[8] M. Yamagiwa et al., Phys. Scr. T145, 014032 (2011).

[9] K. Ohya, Nucl. Instrum. Methods B 153, 58 (1999).

[10] M.E. Woods and B.J. Hopkins, J. Phys. D: Appl. Phys. 20, 1136 (1987).

[11] J.M. Pedgley and G.M. McCracken, Plasma Phys. Control. Fusion 35, 397 (1993)

[12] Y. Nakamura, M. Yamagiwa, T. Kaneko et al., J. Nucl. Mater. 438, S1036 (2013).

[13] N. Ohno, Y. Nakamura, T. Kaneko et al., Physica Scripta 159, 014053 (2014). 